\title{
ANALYSE
}

Die vollzogene Transformation.

Polen nach dem Zusammenbruch der UdSSR vor 30 Jahren

Agnieszka Legucka, Polnisches Institut für Internationale Angelegenheiten,

Warschau

- UMFRAGE

Bewertungen der Systemtransformation in Polen

CHRONIK

07. - 20. September 2021

$$
\begin{aligned}
& \text { Deutsches } \\
& \text { Polen-Institut }
\end{aligned}
$$




\section{Die vollzogene Transformation. Polen nach dem Zusammenbruch der UdSSR vor 30 Jahren}

Agnieszka Legucka, Polnisches Institut für Internationale Angelegenheiten, Warschau

DOI: $10.31205 /$ PA.280.01

\section{Zusammenfassung}

In Polen beschränkt sich die Beurteilung des Zerfalls der Sowjetunion vor 30 Jahren nicht auf das Geschehen Ende des Jahres 1991, als die UdSSR aufhörte, als Subjekt des internationalen Rechtes zu existieren. Es lassen sich mindestens zwei Arten unterscheiden, wie der Zusammenbruch eingeordnet wird: erstens als Zusammenbruch des Kommunismus und zweitens mit Blick auf die innenpolitischen Folgen für Polen (Änderung des politischen und wirtschaftlichen Systems, Veränderungen im kulturellen Bereich) sowie für die Außenpolitik (eine neue Öffnung als vollständig souveräner Staat). Während der Zusammenbruch der Sowjetunion und seine Folgen in ersterem Fall positiv bewertet werden, wurden die Systemveränderungen in Polen in den letzten Jahren kontrovers diskutiert.

\section{Die Beurteilung des Zusammenbruchs der Sowjetunion}

Die Polen betrachten den Zusammenbruch des Kommunismus, der symbolisch mit der Auflösung der UdSSR endete, teilweise als ihren eigenen Erfolg und bewerten ihn eindeutig positiv. Sie identifizieren sich mit der Aussage des tschechischen Schriftstellers Milan Kundera, dass wir »ein vom Osten entführter Teil des Westens" gewesen sind. Die polnische Gesellschaft ist überzeugt, dass die Polen eine große Rolle beim Zerfall des sowjetischen Systems gespielt haben. Gedacht wird hier an die Massenproteste der polnischen Bevölkerung, die oppositionellen Aktivitäten der Unabhängigen Selbstverwalteten Gewerkschaft Solidarność (NSZZ Solidarność) oder den polnischen Papst Johannes Paul II., der die Überzeugung vertrat, dass Europa "mit beiden Lungenflügeln", dem östlichen und dem westlichen, atmen müsse, um vollständig Europa zu sein. Die Polen sind davon überzeugt, dass die Verhängung des Kriegsrechts im Jahr 1981 und schließlich die friedliche Transformation, vorbereitet durch die Verhandlungen am Runden Tisch im Jahr 1989, Teil eines "Dominos" waren, das mit den sog. Vereinbarungen von Minsk und der Auflösung der UdSSR im Dezember 1991 endete. Die Polen erkannten, dass die ungerechte Teilung Europas in einen sowjetischen und einen westlichen Block aufgehoben wurde und die Auflösung der 1945 in Potsdam und Jalta vereinbarten geopolitischen Ordnung neue Möglichkeiten für Polen eröffnete, das nun unabhängig von Moskau und vollständig souverän Entscheidungen im Namen seiner Bürger treffen konnte. Im Bewusstsein der Polen handelt es sich um einen Wendepunkt in der neuesten Geschichte ihres Landes, auf den sie stolz sein können: Im Rahmen des Projektes "Unabhängiges [Polen«, d.Übers.] im Jahr 2016 nannten die Befragten die Systemtransformation, die mit dem Zusammenbruch des kommunistischen Systems assoziiert wird, an zweiter Stelle derjenigen Ereignisse in der Geschichte des polnischen Staates, die die Polen am meisten mit Stolz erfüllen.

\section{Folgen für die Innenpolitik}

Der Zerfall der UdSSR beschleunigte innere Prozesse in Polen, die zu politischen Systemveränderungen sowie einer wirtschaftlichen und sozialen Transformation führten. Die Einführung des demokratischen Systems fand nicht ohne die für Staaten in Transformationsphasen typischen Probleme statt, das sind die Zersplitterung der Parteienlandschaft und Chaos. Letztlich sind daraus die zwei aktuell stärksten Parteien bzw. Gruppierungen hervorgegangen, und zwar Recht und Gerechtigkeit (Prawo i Sprawiedliwość - PiS) und die Bürgerkoalition (Koalicja Obywatelska - KO), deren Rivalität mit einer deutlichen gesellschaftlichen Polarisierung einhergeht. Die Einsetzung demokratischer Institutionen, die die Freiheit des Wortes, eine transparente Justiz und die Bekämpfung der Korruption garantieren, hatten viele positive Folgen für den Alltag der Polen. Obwohl in der letzten Zeit zunehmend Kritik aufkam, dass die Demokratie eingeschränkt werde, und es Kontroversen um die Rechtsstaatlichkeit in Polen gibt, schlägt keine politische Partei in Polen vor, das politische System zu ändern und von der Demokratie abzurücken. Vermutlich aus diesem Grunde ist Polen immer noch ein »free state«, so die Bewertung der internationalen Nichtregierungsorganisation Freedom House. Laut »Democracy Index" sinkt indessen der Grad der polnischen Demokratie (2006: 7,30; 2020: 6,85). Das fügt sich einerseits in den globalen Trend ein, dass sich das Demokratieniveau weltweit verschlechtert, was mit der zunehmenden Attraktivität populistischer Politiker und der Schwächung der Bedeutung der Redefreiheit einhergeht (der 
"World Press Freedom Index" fiel im Jahr 2021 um drei Punkte im Vergleich zu 2019). Andererseits handelt es sich auch um eine Folge des wachsenden Ungleichgewichtes zwischen den gesellschaftlichen Erwartungen in Polen und den Zweifeln an der liberalen Demokratie.

Wie lässt sich nun die Unterstützung der Polen für die Transformation erklären? Aus Umfragen, die das staatliche Meinungsforschungsinstitut CBOS (Centrum Badania Opinii Społecznej) im Mai 2019 durchgeführt hat, ergibt sich, dass 81 Prozent der Polen der Ansicht sind, dass es richtig war, das System zu ändern (siehe Grafik 2 auf S. 7). Davon ist die Mehrheit (52 Prozent) eindeutig überzeugt. Vier Prozent meinen, dass es sich nicht gelohnt hat, die Systemtransformation durchzuführen; 15 Prozent konnten es nicht beurteilen. Ähnlich war es schon in den Umfragen 2009 und 2010, als die Akzeptanz für die Systemveränderungen 82 bzw. 83 Prozent betrug. Nach den Daten von $C B O S$ fiel die Zustimmung für die Systemtransformation in Polen nie unter 56 Prozent. Deren Folgen wurden am negativsten Mitte der 1990er Jahre beurteilt, als sich die Gesellschaft mit ökonomischen Problemen infolge einer Arbeitslosenquote auf Rekordniveau und großer Inflation konfrontiert sah, sowie in den Jahren 2001 bis 2003, als der Beitrittsprozess zur Europäischen Union mit wichtigen, aber gesellschaftlich kostspieligen Reformen erkauft wurde. Mit dem EUBeitritt im Jahr 2004 kam es zu einem Anstieg der positiven Beurteilungen zum Thema Systemtransformation. Diese war nun abgeschlossen und für die Polen ergaben sich neue wirtschaftliche Möglichkeiten. Im Jahr 2019 sagten 68 Prozent, dass die Veränderungen in Polen nach 1989 den Menschen mehr Vor- als Nachteile gebracht hätten, fünf Prozent waren gegenteiliger Meinung (siehe Grafik 3 auf S. 8). Unter denjenigen, die die Transformation positiv bewerten, sehen sechs Prozent ausschließlich Vorteile; bei denjenigen, die sie negativ beurteilen, nehmen nur 0,3 Prozent ausschließlich Nachteile wahr. Hier ist hervorzuheben, dass sich die von $C B O S$ gestellte Frage »Hat es sich gelohnt, das System zu ändern?« nicht so sehr auf die Transformation als weit gefasstes Phänomen bezog, das die Schaffung neuer Regeln in Politik, Wirtschaft und Gesellschaft umfasst sowie den politischen Institutionalisierungsprozess und das Streben nach liberaler Demokratie und Marktwirtschaft meint. Vielmehr zielte die Frage auf die Veränderung des kommunistischen Systems zu einem demokratischen System, ohne auf die sich daraus ergebenden Folgen explizit hinzuweisen. Bestimmte Aspekte der Transformation sowie ihre gesellschaftlichen Implikationen wurden von weiteren Fragen abgedeckt. Allerdings sollte das Bild der positiven Beurteilung über den Zusammenbruch des Kommunismus nicht mit der Bewertung der Systemtransformation in Polen verwechselt werden, denn dieses Bild gestaltet sich sehr viel differenzierter.

\section{Folgen für die Außenpolitik}

Mit dem Zerfall der UdSSR erlangte Polen vollständige Unabhängigkeit und Souveränität und konnte beginnen, seine nationalen Interessen auf der internationalen Bühne selbständig zu verfolgen. Die Polen sind sich bewusst, dass es ohne die Schwächung des östlichen Nachbarn nicht zur Loslösung aus der Abhängigkeit von Moskau gekommen wäre und - was damit einhergeht - nicht zum Beitritt zur NATO und EU. Die Veränderung der internationalen Situation, der Zusammenbruch der UdSSR, die Erweiterung der NATO und die Intensivierung der Integration der EU schufen für Polen gute Bedingungen. Die westliche Orientierung in der polnischen Außenpolitik war u. a. auf die Zusammenarbeit mit Deutschland als größtem Wirtschaftspartner und einflussreichstem Staat in der Europäischen Union ausgerichtet. Anfang der 1990er Jahre hatten die Polen Angst vor dem wiedervereinigten Deutschland, aber schon bald wurde die Notwendigkeit hervorgehoben, einen Partner für den polnischen Weg zur europäischen Integration zu suchen. Sowohl unter dem Einfluss der wirtschaftlichen als auch der gesellschaftlichen Kontakte verbesserte sich über die Jahre das Bild der Deutschen in Polen. Im Jahr 1993 nahmen in einer CBOSUmfrage 23 Prozent die Deutschen positiv wahr, im Jahr 2019 bereits 43 Prozent. In den letzten Jahren jedoch bewerten die Polen die Beziehungen zu den Deutschen als schlecht. Beeinflusst wird dies von gegensätzlichen Interessen der polnischen und der deutschen Regierung bei den Themen Nord Stream 2, Haltung zur europäischen Integration sowie in Sachen Rechtsstaatlichkeit.

In der Ostpolitik stützt sich die polnische Staatsräson auf drei fundamentale Interessen: Erstens die Achtung und Bewahrung der Souveränität und territorialen Integrität der östlichen Staaten, zweitens die Begrenzung der imperialen Politik Russlands und drittens die Demokratisierung und Europäisierung der östlichen Staaten. Der gemeinsame Nenner dieser Interessen ist, den Erhalt und die Entwicklung des polnischen Staates zu sichern. Gegenüber den Staaten der Östlichen Partnerschaft (Armenien, Aserbaidschan, Belarus, Georgien, Moldau, Ukraine) war das Hauptziel der polnischen Ostpolitik, die Stabilität, Souveränität und territoriale Integrität dieser Staaten angesichts des wachsenden Drucks und im nächsten Schritt der militärischen Aggression von Seiten Russlands zu unterstützen. Aus diesen Gründen legte die polnische Diplomatie im Rahmen ihrer EU-Mitgliedschaft zunächst den Schwerpunkt auf die Demokratisierung und Europäisierung der Staaten der Östlichen Partnerschaft. Sie war Befürworterin einer Öffnung der EU und zeigte sich überzeugt, dass nur die Perspektive einer EU-Mitgliedschaft eine wirksame Methode ist, damit sich die Staaten in Richtung europäischer Standards reformieren. Dies 
sollte vor allem die Ukraine, Moldawien und Georgien in politische und wirtschaftliche Modernisierungsprozesse unter dem Schild der EU einbinden. Gleichzeitig sollte es dazu beitragen, dass die Zone in Europa, in der Stabilität herrscht, erweitert wird, sowie die Sicherheit Polens vergrößern. Da sich bei der EU-Erweiterung nach Osten bisher kein Erfolg abzeichnete und auch die Fortschritte bei den Reformen in den Staaten der Östlichen Partnerschaft nur mäßig sind, hat sich die polnische Diplomatie in den Jahren 2015 bis 2020 vor allem darauf konzentriert, die äußere Sicherheit in Hinblick auf Russland sicherzustellen und die Lage jenseits der polnischen Ostgrenze zu stabilisieren. Eine Priorität wurde die Stärkung der NATO-Ostflanke und die entschiedene Fürsprache für Sanktionen gegenüber Russland. Die Haltung zu Souveränität und territorialer Integrität wird von der Europäischen Union unterstützt. Als Reaktion auf die Annexion der Halbinsel Krim im Jahr 2014 durch Russland, die von Polen nicht anerkannt wurde und auf die die EU mit Sanktionen gegenüber den verantwortlichen Russen antwortete, verfolgt die EU ähnlich wie Polen die Politik, durch Aggression herbeigeführte Grenzveränderungen in Europa nicht anzuerkennen. Gleichzeitig veranlassten die aggressive Politik Russlands und sein Einsatz von Gewalt in den internationalen Beziehungen die polnische Regierung, den Kleinen Grenzverkehr zur russischen Oblast Kaliningrad im Jahr 2016 zu schließen, obgleich ihm die polnischen lokalen Behörden positiv gegenüber standen, da er ihnen seit dem Jahr 2012 ökonomische Vorteile einbrachte.

Die PiS-Regierung legte größeres Gewicht auf historische Fragen, im Falle der Ukraine insbesondere auf divergierende Bewertungen des Massakers von Wolhynien und der Tätigkeit der Ukrainischen Aufständischen Armee (1943/44) und bezogen auf Russland auf das Gedenken des Verbrechens von Katyn (1940). Polen kritisierte, dass der Oberste Rat der Ukraine im April 2015 das Gesetzespaket zur sog. Entkommunisierung verabschiedet hat, das vom Ukrainischen Institut für Nationales Gedenken vorbereitet worden war. Eines der Gesetze erkennt die Mitglieder der Ukrainischen Aufständischen Armee als Kämpfer für die Unabhängigkeit der Ukraine an und sieht vor, diejenigen juristisch zur Verantwortung zu ziehen, die die Unabhängigkeitskämpfer öffentlich herabwürdigen oder die Legalität ihrer Tätigkeiten in Frage stellen.

Der Zusammenbruch der UdSSR wurde nicht zur Grundlage für gutnachbarliche Beziehungen zwischen Polen und Russland. Die Polen hörten nicht auf, Russland als Bedrohung wahrzunehmen, was sich 2014 noch verstärkte. Polen machte auf das Ausbaupotential im Westlichen Militärbezirk Russlands und die fortschreitende Militarisierung der Oblast Kaliningrad auf- merksam, wo neue Waffensysteme stationiert werden, u. a. Mittelstreckenraketen, die mit Nuklearsprengköpfen ausgestattet werden können. Polen erklärte, dass es die russische Vision einer politischen Ordnung in Europa nicht akzeptiert und der Einteilung des Kontinents in Einflusszonen nicht zustimmen wird. Über die Bedrohung von Seiten Russlands als Hauptgefahr für Polen kann man im "Verteidigungskonzept der Republik Polen« vom Mai 2017 lesen. Dort heißt es: "[...] wir sind überzeugt, dass Russland bis zum Jahr 2032 eine aggressive Außenpolitik betreiben [...] und die Hauptursache für Instabilität in der Nachbarschaft der NATO-Ostflanke bleiben wird." Diese Einschätzungen wurden in der "Nationalen Sicherheitsstrategie« vom Mai 2020 wiederholt, in der unterstrichen wurde, dass »die Russische Föderation auch Aktivitäten unterhalb der Ebene des Krieges (hybride Aktivitäten) unternehmen wird, die das Risiko bergen, dass es zum Ausbruch eines Konfliktes kommt". Ende 2019 vertrat der russische Präsident Wladimir Putin falsche Thesen über die Beteiligung Polens am Ausbruch des Zweiten Weltkrieges und bezichtigte die Polen der Zusammenarbeit mit Hitler. Dies war ein weiteres Kapitel im Streit über die Geschichte mit Polen. Gründe für dieses Vorgehen Russlands gab es einige: Erstens handelte es sich um eine Reaktion auf die von der polnischen Regierung betriebene Demontage von Denkmälern, der sog. Monumente der Dankbarkeit gegenüber der UdSSR auf polnischem Territorium. Zweitens war es eine Antwort auf die Resolution des Europäischen Parlamentes, das sich auf Antrag Polens im September 2019 über die Bedeutung des europäischen historischen Gedächtnisses abgestimmt und an den Hitler-Stalin-Pakt sowie an die aktuelle Infragestellung des Vertrages zwischen den Nationalsozialisten und den Kommunisten durch die russischen Machthaber erinnert hat.

\section{Folgen für die Wirtschaft}

Das Wohlstandsniveau gemessen am Bruttoinlandsprodukt (BIP) pro Kopf nach Kaufkraftparität ist in Polen heute fast fünf Mal so hoch wie vor 30 Jahren und die reale Kaufkraft des BIP pro Kopf hat sich verdreifacht. Aus dem Blickwinkel der statistischen Daten erwies sich die Transformation in Polen als wirtschaftlicher Erfolg, doch ihre sozialen und politischen Folgen sind vielschichtig. Im Vergleich zu allen anderen Ländern des sowjetischen Blocks entwickelte sich die polnische Wirtschaft nach dem Zusammenbruch des Realsozialismus am schnellsten. Daher äußern sich die Polen mehrheitlich zufrieden über die wirtschaftliche Situation im Vergleich zur Zeit des Kommunismus. Gleichzeitig kam es mit dem zunehmenden Wohlstand der Polen zur ökonomischen Ausdifferenzierung. Am zufriedensten waren die gut gebildeten Bürger mit den Veränderungen, meistens Einwohner von Großstädten. 
Die Reformen zur Überführung der Planwirtschaft in eine freie Marktwirtschaft wurden im Rahmen der sog. Schocktherapie und nach dem Plan des damaligen Finanzministers Leszek Balcerowicz durchgeführt. Die grundsätzlichen Probleme, mit denen sich Polen zu Beginn der Transformation konfrontiert sah, waren die Hyperinflation, das ineffiziente politisch instrumentalisierte Produktionssystem sowie die Dominanz der staatlichen Produktion. Mit Hilfe von Privatisierungen gelang es, die staatlichen Betriebe zu ersetzen. In den Jahren 1990 bis 2004 belief sich der Gesamtwert des in Polen in Form von Direktinvestitionen angelegten ausländischen Kapitals auf 84,5 Mrd. US-Dollar. Nur ein Teil davon war für den Kauf der existierenden Unternehmen bestimmt. Auf dem Höhepunkt erreichte der Anteil des ausländischen Kapitals bei den Einnahmen aus der Privatisierung 82 Prozent; im Jahr 2004 ist er auf neun Prozent gesunken. Der Prozess der Eigentumsumwandlung, der die Kommerzialisierung in Gang setzte, betraf zwischen 1990 und 20046.002 staatliche Unternehmen, d. h. 71 Prozent ihres Bestands im Jahr 1990. Eine der sozialen Folgen der Privatisierung war der Anstieg der Arbeitslosigkeit.

Untersuchungen zeigen, dass die Polen immer noch den Eindruck haben, dass das Lebensniveau in Polen niedriger ist als in den westlichen Ländern. Auch negative Erinnerungen aus den 1990er Jahren werden sich in das Gedächtnis der über 44-Jährigen eingeprägt haben. So zeigten sich die Einwohner kleinerer Städte und Dörfer und die geringer Gebildeten in Umfragen am wenigsten zufrieden. Die Erfahrung der schwierigen Transformationsphase legte sich bei einem Teil der polnischen Gesellschaft auf die politische Stimmung.

\section{Die Kultur 30 Jahre nach dem Zusammenbruch der UdSSR}

Das erste Jahrzehnt der Transformation war schwierig für die Kulturschaffenden, denn die Regierungen reduzierten die finanziellen Mittel für diesen Bereich. Maßnahmen, die bei der Dezentralisierung und Entpolitisierung der Kultur helfen und diese mit den Erfordernissen des Marktes kompatibel machen sollten, machten es notwendig, Kompetenzen und Pflichten von der Zentralebene auf die regionale und lokale Ebene zu verlagern, so dass den Woiwoden und Stadtpräsidenten Aufgaben im kulturellen Bereich übertragen wurden. Im März 2000 wurde in Warschau das Adam Mickiewicz-Institut eröffnet, das erste staatliche Institut, das die polnische Kultur im Ausland verbreitet und fördert. Die entscheidende positive Rolle für die öffentlichen Kulturinstitutionen spielte jedoch die Europäische Union. Mit Hilfe finanzstarker Strukturfonds konnten viele Renovierungen durchgeführt und neue Objekte gebaut werden. So entstanden das Museum des Zweiten Weltkrie- ges (Muzeum II Wojny Światowej) in Danzig (Gdańsk), das Nationale Musikforum (Narodowe Forum Muzyki) in Breslau (Wrocław), das Museum für Zeitgenössische Kunst (Muzeum Sztuki Współczesnej) in Krakau (Kraków), das Zentrum für Begegnungen der Kulturen (Centrum Spotkania Kultur) in Lublin oder die Stettiner Philharmonie (Filharmonia Szczecinsska). Zusätzlich wurde das kulturelle Leben mehrerer Städte durch die Bewerbung für die Europäische Kulturhauptstadt angefacht. In der Überzeugung, dass die Kultur für den Aufbau der nationalen Identität von großer Bedeutung ist, hat die PiS-Regierung die Aufwendungen für den kulturellen Bereich um 34 Prozent erhöht. Trotz einer gewissen Kommerzialisierung überwiegt auch nach 30 Jahren die öffentliche Finanzierung der Kultur durch den Staatshaushalt und die Selbstverwaltungsbehörden die privaten Mittel. Im Jahr 2019 betrugen die öffentlichen Ausgaben für die Kultur 0,5 Prozent des BIP. Gleichzeitig nimmt die Bedeutung zusätzlicher öffentlicher Finanzierungsmittel zu, beispielsweise Gelder der Europäischen Union, Einnahmen der staatlichen Lotterie o. ä.

\section{Systemtransformation - die aktuelle Debatte}

In Polen findet in den letzten Jahren eine Diskussion über die Bewertung der Systemtransformation statt. Obgleich die deutliche Mehrheit der Polen eine positive Bilanz über die Veränderungen zog, kamen nach der Wirtschaftskrise 2008/09 Stimmen auf, dass Polen die Zeit nach dem Zusammenbruch der UdSSR nicht angemessen genutzt habe. Seit 2013 ließ sich eine zunehmende Kritik am Systemwechsel feststellen. 2013 überwog die Auffassung (59 Prozent), dass man mehr hätte erreichen können und dass die Chance, die die Systemveränderungen geboten haben, nicht ausreichend genutzt wurde. Im politischen Diskurs traten zwei einander entgegen gesetzte Narrationen über die Ereignisse auf. Die eine lautete, dass der Runde Tisch der Sieg der demokratischen Kräfte (der Solidarność und Lech Wałęsas) über die Kommunisten und die Transformation ein politischer und wirtschaftlicher Erfolg war und zur Erlangung des wichtigsten Ziels geführt hat, nämlich Polen in den euroatlantischen Strukturen (EU und NATO) zu verwurzeln. In der Gegennarration überwiegen die kritischen Meinungen zur Transformation. Manche Politiker - vor allem aus der PiS - meinen, dass die Verhandlungen am Runden Tisch das Ergebnis eines Kompromisses zwischen einem Teil der Solidarność-Angehörigen und den Kommunisten waren, wofür die fehlende "Durchleuchtung" der kommunistischen Vergangenheit (die sog. Lustration) sowie die fehlende Abrechnung mit der Vergangenheit sprächen. Lech Wałęsa wurde der Zusammenarbeit mit dem kommunistischen Geheimdienst der Volksrepublik ange- 
klagt und die demokratischen Kräfte übergaben die Regierungsverantwortung den Postkommunisten, als die Demokratische Linksallianz (Sojusz Lewicy Demokratycznej-SLD) die Parlamentswahlen 1997 gewonnen hatte, weil es so am Runden Tisch verabredet worden sei. In den Äußerungen der Politiker überwogen kritische Meinungen zum Verlauf der Transformation, insbesondere zu ihren Kosten (im Allgemeinen bezieht sich das den Balcerowicz-Plan), sowie zu den Haupakteuren (Streit um Lech Wałęsa). Obgleich diese Narration nur geringfügig die Einstellungen der öffentlichen Meinung beeinflusst, je nach individueller Situation und den Transformationserfahrungen desjenigen, der sich in Umfragen dazu äußert, kann sie in der Zukunft die dominierende Erzählung werden.

\section{Schlussfolgerungen}

Der Zusammenbruch der UdSSR wird in Polen eindeutig positiv beurteilt. Die Systemtransformation ist eine der wichtigsten Erfahrungen des 20. Jahrhunderts, die sich in das kollektive Gedächtnis der Polen eingeschrieben haben. Sie wird von den Polen aber vor allem als Zerfall des Kommunismus verstanden. Die Polen betrachten den Moment als Wendepunkt für die innenpolitische und außenpolitische Lage des Staates, als Beginn der Demokratisierung des öffentlichen Lebens, Beendigung der Korruption, Entwicklung der Zivilgesellschaft, Eintritt des polnischen Staates in die Strukturen internationaler Organisationen wie der NATO und der Europäischen Union. All dies hat den Alltag der Polen positiv beeinflusst. Dass es richtig war, die Transformation zu vollziehen, ruft in der polnischen Gesellschaft aktuell keinen Widerspruch hervor. Die deutliche Mehrheit der Polen steht den Veränderungen Ende der 1980er, Anfang der 1990er Jahre positiv gegenüber. Indessen fängt es an, dass in Polen die Folgen der Transformation unterschiedlich interpretiert werden. Die Systemveränderungen und deren Bewertungen spalten die Polen. Die oben angeführten Untersuchungen zeigen, dass diese Prozesse gegensätzliche Meinungen in der polnischen Gesellschaft hervorrufen sowie Gegenstand des Streites im öffentlichen Diskurs über die Transformation sind. Dies trägt zur wachsenden gesellschaftlichen Polarisierung und zum Wettbewerb der Narrationen bei, ob Polen die letzten 30 Jahre der Transformation genutzt hat bzw. ungenutzt ließ.

Übersetzung aus dem Polnischen: Silke Plate

\section{Über die Autorin}

Dr. habil. Agnieszka Legucka ist wissenschaftliche Mitarbeiterin mit Schwerpunkt Russland im Osteuropa-Programm des Polnischen Instituts für Internationale Angelegenheiten (Polski Instytut Spraw Międzynarodowych - PISM), Warschau. Ihre Forschungsgebiete sind die Innen- und Außenpolitik Russlands, die Beziehungen Russlands zur Europäischen Union und zur NATO, Desinformationsaktivitäten und hybride Gefahren von Seiten Russlands sowie Fragen der Sicherheit und Konflikte in der östlichen Nachbarschaft der Europäischen Union. Außerdem ist sie als außerordentliche Professorin am Fachbereich Finanzen und Internationale Beziehungen der Akademie für Finanzen und Business Vistula (Akademia Finansów i Biznesu Vistula), Warschau, sowie stellvertretende Chefredakteurin der Zeitschrift Sprawy Międzynarodowe (»Internationale Angelegenheiten«) tätig.

\section{Lesetipps}

- Stanisław Gomułka, Poland's economic and social transformation 1989-2014 and contemporary challenges, Central Bank Review, Volume 16, Issue 1, March 2016, p. 19-23.

- Bartosz Rydliński, Economic Crisis as a Factor of the Neoliberal Policy in Poland, Prakseologia nr 159/2017, p. 39-62. 


\section{Bewertungen der Systemtransformation in Polen}

Grafik 1: Die wirtschaftliche Situation ist heutzutage für die meisten Menschen besser als zur Zeit des Kommunismus (\%, Ländervergleich)

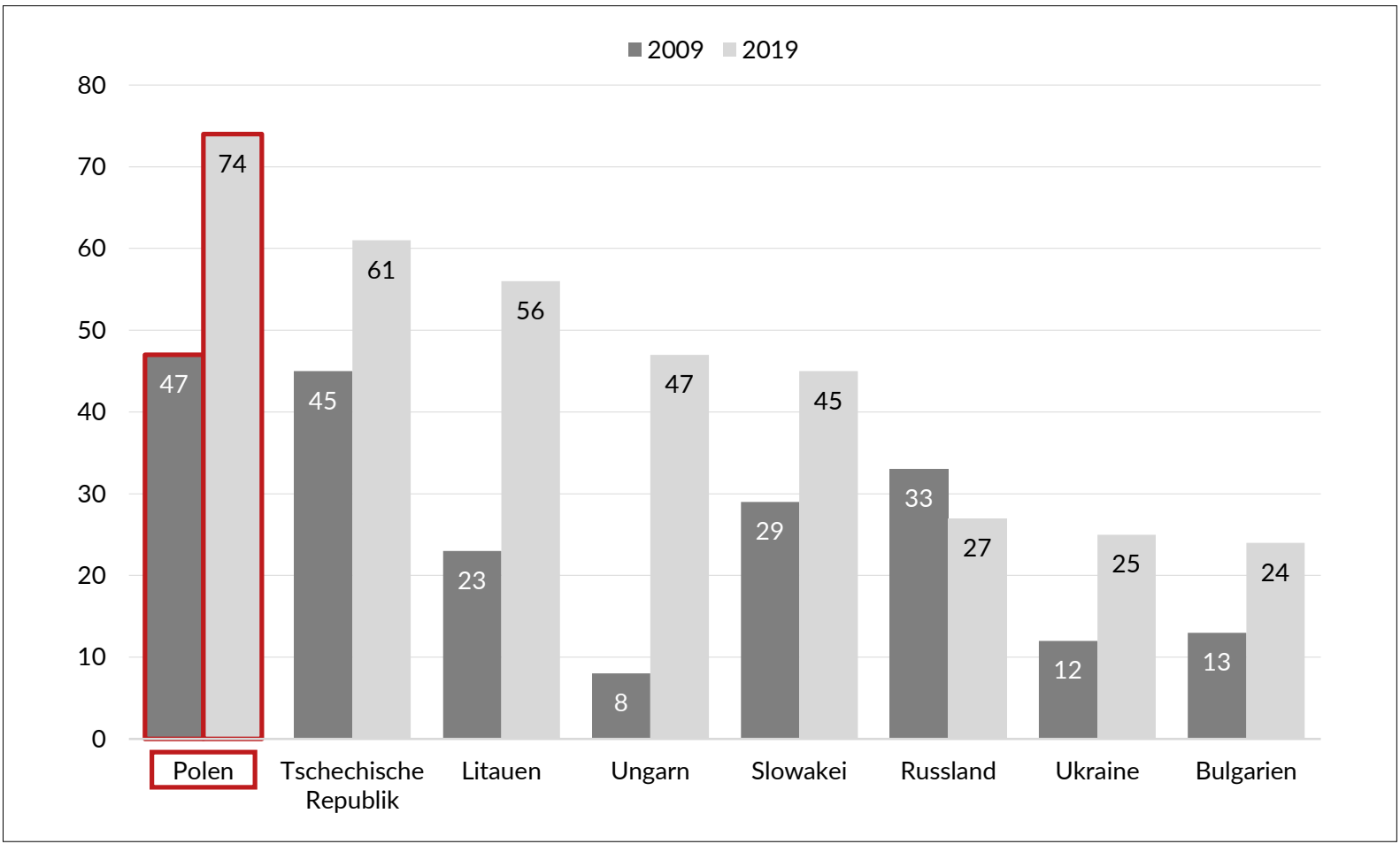

Quelle: Pew Research Center, 14.10.2019. https://www.pewresearch.org/global/2019/10/14/political-and-economic-changes-since-the-fall-ofcommunism/pg_10-15-19-europe-values_hungary/ (abgerufen am 16.09.2021).

Grafik 2: Können Sie aus der Rückschau sagen, dass es sich gelohnt hat/nicht gelohnt hat, 1989 das System in Polen zu verändern? (\%, Jahresvergleich)

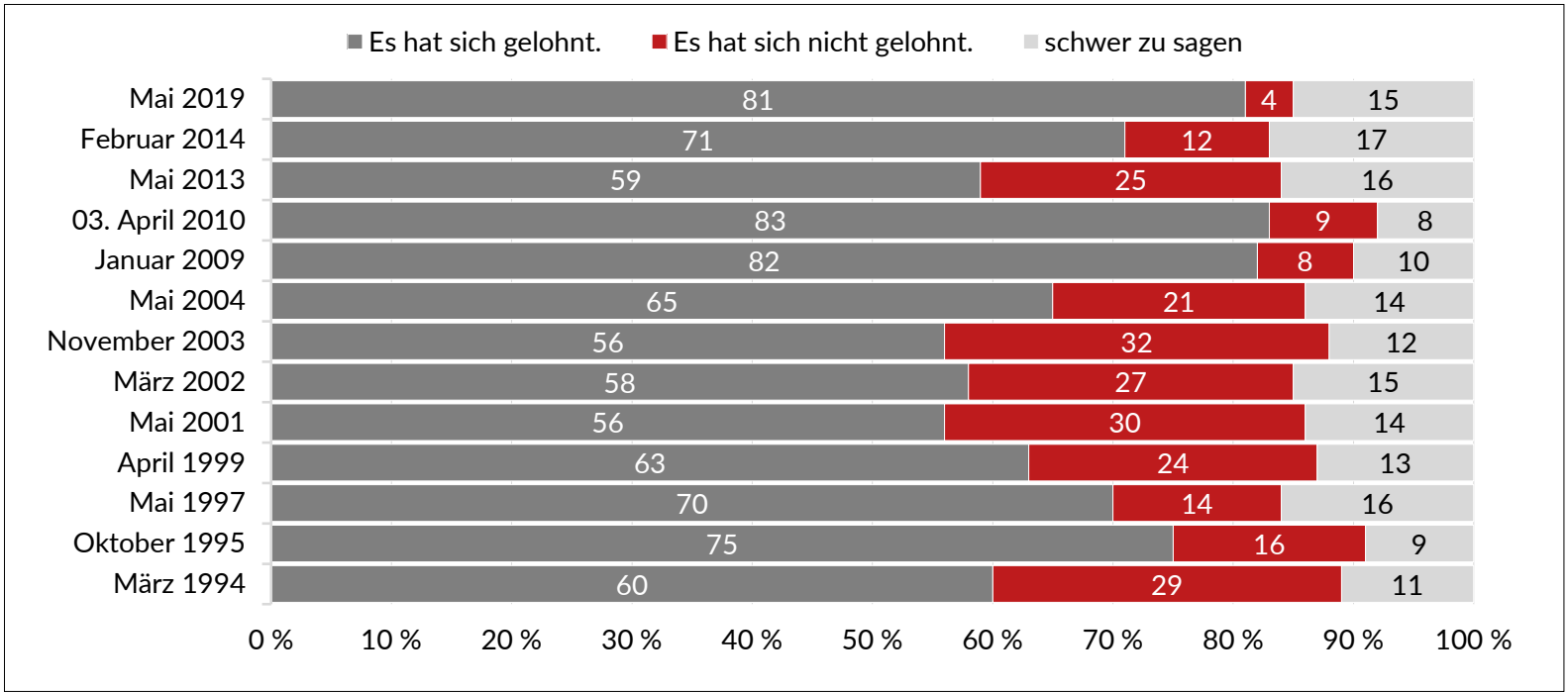

Quelle: CBOS: Komunikat z badań 76/2019: Czy warto było zmieniać ustrój? Ocena przemian po 1989 roku [Hat es sich gelohnt, das System zu verändern? Die Bewertung der Veränderungen nach 1989]. Warszawa 06/2019. www.cbos.pl 
Grafik 3: Haben die Veränderungen, die in Polen ab 1989 stattfanden, den Menschen mehr Vorteile oder mehr Nachteile gebracht? (\%, Jahresvergleich)

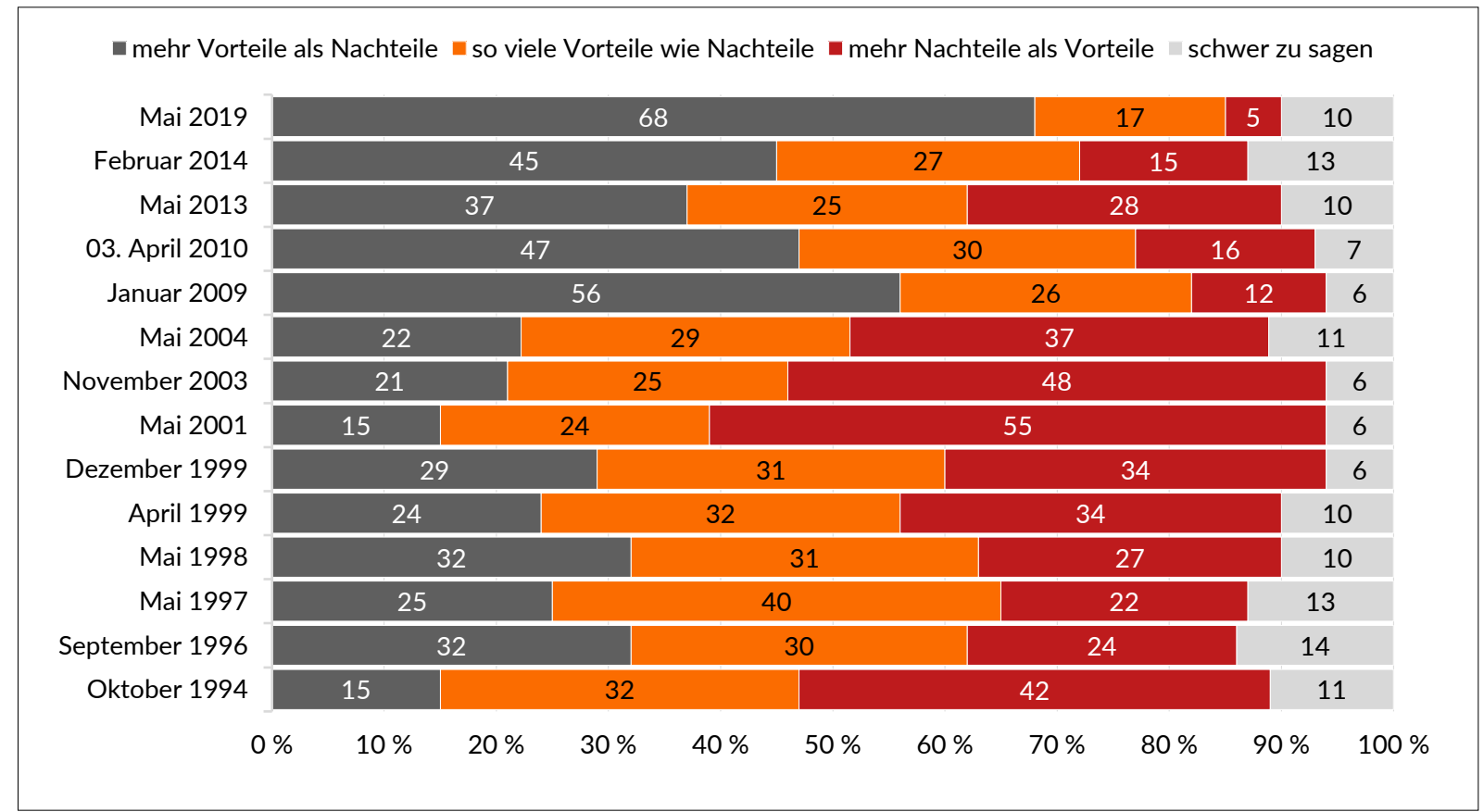

Quelle: CBOS: Komunikat z badań 76/2019: Czy warto było zmieniać ustrój? Ocena przemian po 1989 roku [Hat es sich gelohnt, das System zu verändern? Die Bewertung der Veränderungen nach 1989]. Warszawa 06/2019. www.cbos.pl

Grafik 4: Haben die Veränderungen, die in Polen ab 1989 stattfanden, den Menschen mehr Vorteile oder mehr Nachteile gebracht? (\%, Altersgruppen, 2019)

mehr Vorteile als Nachteile mo viele Vorteile wie Nachteile $\square$ mehr Nachteile als Vorteile schwer zu sagen

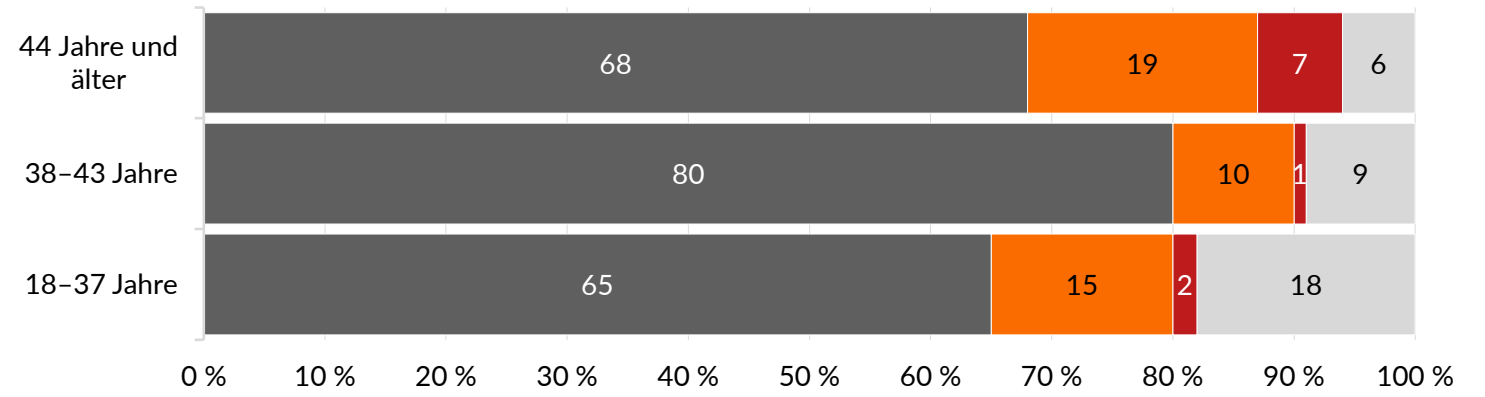

Quelle: CBOS: Komunikat z badań 76/2019: Czy warto było zmieniać ustrój? Ocena przemian po 1989 roku [Hat es sich gelohnt, das System zu verändern? Die Bewertung der Veränderungen nach 1989]. Warszawa 06/2019. www.cbos.pl 


\section{Grafik 5: Von welchem Ereignis lässt sich sagen, dass es das Ende des kommunistischen Systems in Polen} markiert? (\%)

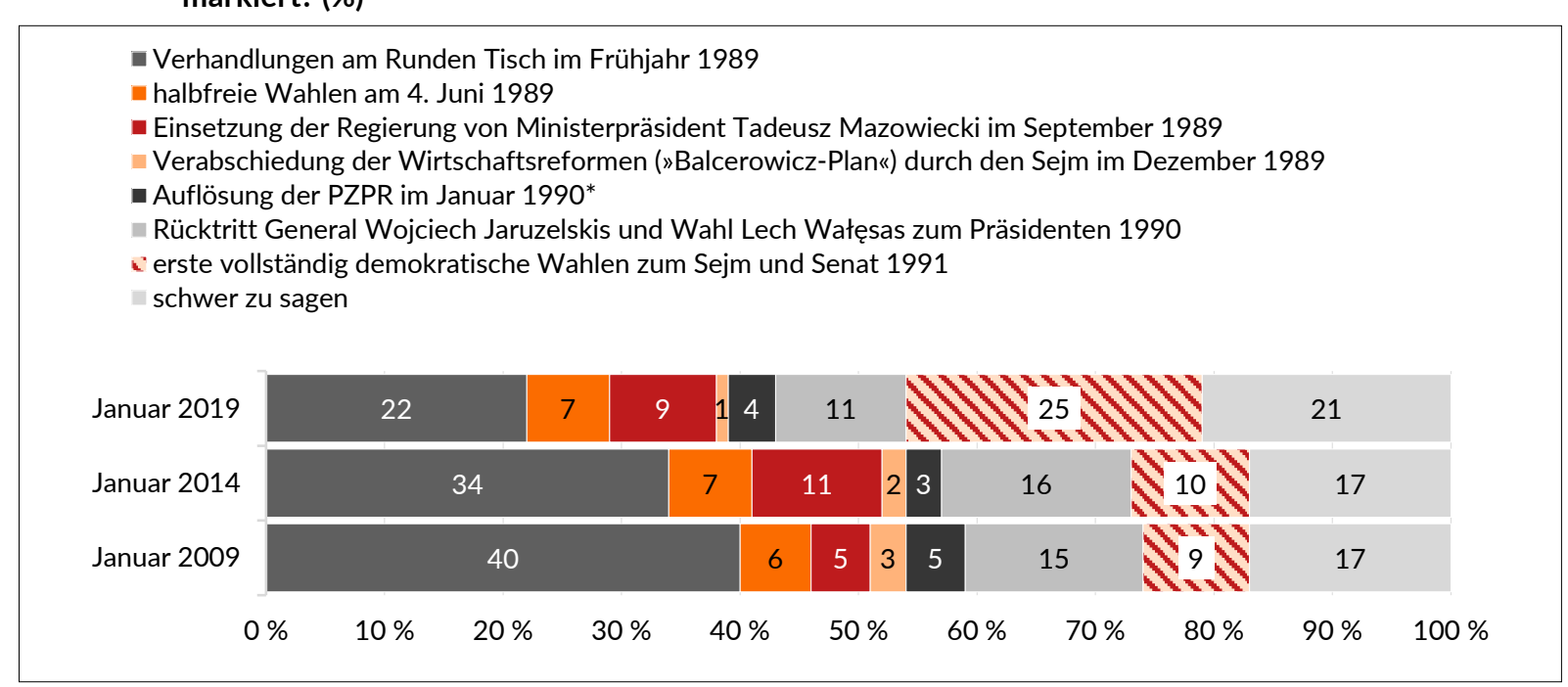

*PZPR - Polska Zjednoczona Partia Robotnicza/Polnische Vereinigte Arbeiterpartei

Quelle: CBOS: Komunikat z badań 16/2019: Trzydziesta rocznica obrad Okragłego Stołu [30. Jahrestag der Verhandlungen am Runden Tisch]. Warszawa 02/2019. www.cbos.pl

\section{Grafik 6: Stimmen Sie zu, dass...? (\%, 2019)}

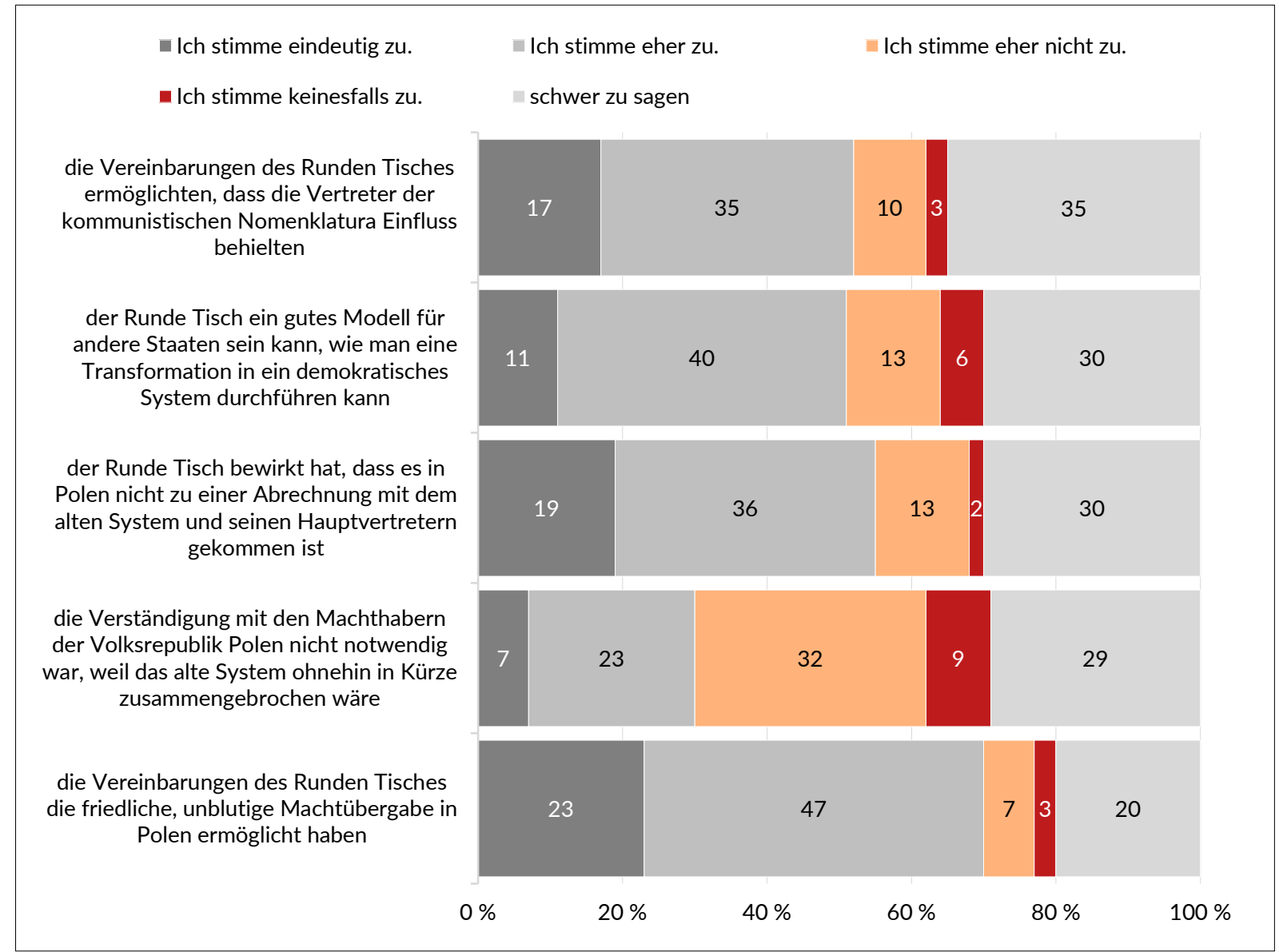

Quelle: CBOS: Komunikat z badań 16/2019: Trzydziesta rocznica obrad Okragłego Stołu [30. Jahrestag der Verhandlungen am Runden Tisch]. Warszawa 02/2019. www.cbos.pl 


\section{Grafik 7: Stimmen Sie zu, dass...? (\%, Jahresvergleich)}

die Vereinbarungen am Runden Tisch die friedliche, unblutige Machtübergabe in Polen ermöglicht haben
- Ich stimme eindeutig zu.
Ich stimme eher zu.
Ich stimme eher nicht zu.
- Ich stimme keinesfalls zu.
schwer zu sagen

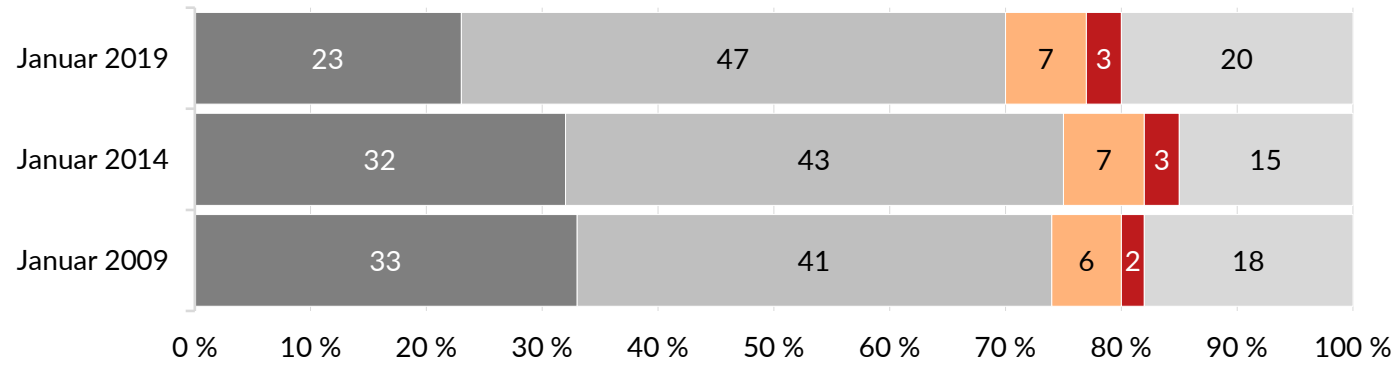

die Verständigung mit den Machthabern der Volksrepublik Polen nicht notwendig war, weil das alte System ohnehin in Kürze zusammengebrochen wäre
- Ich stimme eindeutig zu.
Ich stimme eher zu.
Ich stimme eher nicht zu.
- Ich stimme keinesfalls zu.
schwer zu sagen

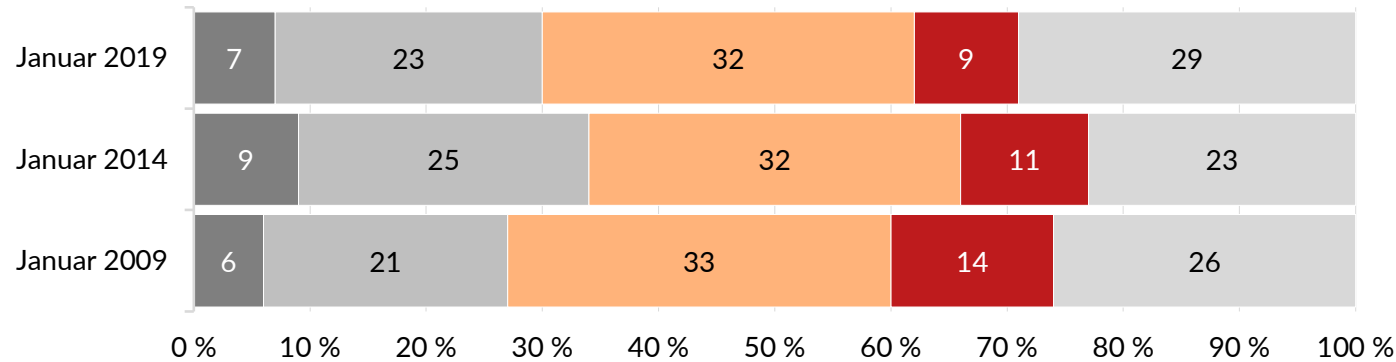

der Runde Tisch bewirkt hat, dass es in Polen nicht zu einer Abrechnung mit dem alten System und seinen Hauptvertretern gekommen ist
- Ich stimme eindeutig zu.
- Ich stimme eher zu.
Ich stimme eher nicht zu.

- Ich stimme keinesfalls zu.

schwer zu sagen

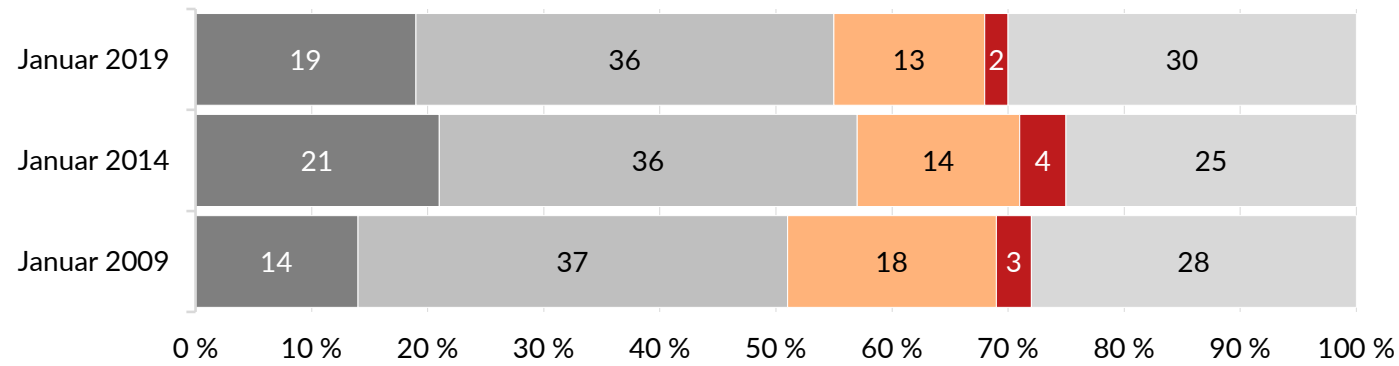

Quelle: CBOS: Komunikat z badań 16/2019: Trzydziesta rocznica obrad Okragłego Stołu [30. Jahrestag der Verhandlungen am Runden Tisch]. Warszawa 02/2019. www.cbos.pl 


\section{7. - 20. September 2021}

\begin{tabular}{|c|c|}
\hline 07.09.2021 & $\begin{array}{l}\text { Die Europäische Kommission beantragt beim Europäischen Gerichtshof (EuGH) finanzielle Sanktionen gegen } \\
\text { Polen. Justizminister Zbigniew Ziobro sagt, das Vorgehen sei ein hybrider juristischer Krieg mit ökonomischen } \\
\text { Implikationen, den die EU gegen Polen führe. Der Hintergrund ist, dass der EuGH Mitte Juli geurteilt hat, } \\
\text { die Disziplinarkammer des Obersten Gerichtes (Sąd Najwyższy - SN) in Polen sei nicht EU-rechtskonform, } \\
\text { so dass ihre Tätigkeit eingestellt werden muss. Polen hat das Urteil jedoch nicht vollständig umgesetzt, da die } \\
\text { Erste Präsidentin des SN, Małgorzata Manowska, Anfang August angeordnet hat, dass die Disziplinarkam- } \\
\text { mer noch alte Fälle abarbeiten soll. }\end{array}$ \\
\hline 08.09 .2021 & $\begin{array}{l}\text { Ministerpräsident Mateusz Morawiecki äußert sich vorsichtig optimistisch, dass sich Polen und die Europäische } \\
\text { Kommission über den polnischen Landesaufbauplan (Krajowy Plan Odbudowy) verständigen werden. Zurzeit } \\
\text { hält die Europäische Kommission ihre Zustimmung zurück, die notwendig ist, damit Beihilfen aus dem Euro- } \\
\text { päischen Wiederaufbaufonds zur Bekämpfung der Folgen der Corona-Pandemie an Polen ausgezahlt werden } \\
\text { können. In Aussicht steht eine Summe von } 36 \text { Mrd. Euro. In der vergangenen Woche signalisierte die Euro- } \\
\text { päische Kommission, dass ihre Weigerung mit dem Konflikt zwischen der EU und Polen über die polnische } \\
\text { Justizreform in Verbindung steht sowie damit, dass Polen den Vorrang des EU-Rechts vor nationalem Recht in } \\
\text { Zweifel zieht. Von Journalisten gefragt, ob er Probleme bei der Auszahlung der EU-Hilfsgelder befürchte, sagt } \\
\text { Morawiecki, wenn in der Europäischen Union Recht und Rechtsstaatlichkeit herrschen, sollten keine Schwie- } \\
\text { rigkeiten befürchtet werden. }\end{array}$ \\
\hline 09.09 .2021 & $\begin{array}{l}\text { Der Senat lehnt die von Recht und Gerechtigkeit (Prawo i Sprawiedliwość - PiS) initiierte Novelle des Rund- } \\
\text { funk und Fernsehgesetzes, die sogenannte Lex TVN, mit } 53 \text { Nein-Stimmen bei } 37 \text { Ja-Stimmen und drei Ent- } \\
\text { haltungen ab. Sie sieht vor, dass Mediengesellschaften mit Sitz in Polen nicht von Konzernen außerhalb des } \\
\text { europäischen Wirtschaftsraumes kontrolliert werden sollen. Kritiker sehen darin einen Angriff auf den regie- } \\
\text { rungskritischen Sender TVN, der zum US-amerikanischen Konzern Discovery Inc. gehört. Das Gesetz geht } \\
\text { nun zurück an den Sejm, der es Anfang August verabschiedet hat. }\end{array}$ \\
\hline 11.09 .2021 & $\begin{array}{l}\text { Bundeskanzlerin Angela Merkel spricht sich bei ihrem Treffen mit Ministerpräsident Mateusz Morawiecki } \\
\text { in Warschau dafür aus, den Streit zwischen Polen und der Europäischen Kommission über die Justizreform } \\
\text { in Polen im Dialog zu lösen. Politik sei mehr, als zu Gericht zu gehen, so Merkel. Zurzeit beschäftigen sich } \\
\text { sowohl das polnische Verfassungstribunal (Trybunał Konstytucyjny - TK) als auch der Europäische Gerichts- } \\
\text { hof (EuGH) mit verschiedenen Aspekten der umstrittenen Reform. }\end{array}$ \\
\hline 13.09 .2021 & $\begin{array}{l}\text { Verteidigungsminister Mariusz Błaszczak und Außenminister Zbigniew Rau nehmen in Riga (Lettland) an einem } \\
\text { Treffen mit ihren Amtskollegen aus Estland, Lettland und Litauen teil. Man habe über gemeinsame Militär- } \\
\text { übungen gesprochen und darüber, wie man gemeinsam die eigene Sicherheit gewährleisten könne, so Błaszczak. } \\
\text { Hintergrund ist der "hybride Konflikt«, den Belarus gegen seine westlichen Nachbarn begonnen hat, und das } \\
\text { aktuell stattfindende russische Militärmanöver "Zapad-21«, an dem u. a. belarussische Truppen teilnehmen. }\end{array}$ \\
\hline 14.09 .2021 & $\begin{array}{l}\text { Witold Kozłowski, Marschall der Woiwodschaft Kleinpolen (województwo małopolskie), kündigt die Beru- } \\
\text { fung eines Beauftragten und eines Rates für Gleichstellung und die Rechte der Familie an. Damit reagiere der } \\
\text { Vorstand der Woiwodschaft auf die Mitteilung der Europäischen Kommission vom Juli 2021, dass EU-Gelder } \\
\text { zurückgehalten werden können, wenn die Woiwodschaft nicht die sogenannte "Anti-LGBT Erklärung«aus } \\
\text { dem Jahr } 2019 \text { zurücknimmt. Im August } 2021 \text { stimmte der Woiwodschaftslandtag (sejmik) gegen den Wider- } \\
\text { ruf. Dem geplanten Rat sollen der Beauftragte für die europäischen Fonds, ein Koordinator für juristische Fra- } \\
\text { gen, Vertreter der Fraktionen im sejmik und Vertreter von zivilgesellschaftlichen Organisationen für Gleichbe- } \\
\text { handlung sowie Familienrechte angehören. }\end{array}$ \\
\hline 14.09 .2021 & $\begin{array}{l}\text { Piotr Pacewicz, Chefredakteur der online-Informationsplattform »OKO.press«, gibt bekannt, dass } 30 \text { Redaktio- } \\
\text { nen einen Aufruf an die Regierung unterzeichnet haben, in dem gefordert wird, dass Medienvertreter Zugang } \\
\text { zu den mit Ausnahmenzustand belegten Gebieten an der polnischen Grenze zu Belarus bekommen. Der Aus- } \\
\text { nahmezustand wurde Anfang September "zum Schutz der öffentlichen Ordnung« verhängt. Der Hintergrund } \\
\text { ist, dass auf der belarussischen Seite der Grenze seit Wochen Flüchtlinge u. a. aus Afghanistan kampieren, die } \\
\text { in die Europäische Union einreisen wollen. Sie wurden vom belarussischen Präsidenten Alexander Lukaschenko } \\
\text { dafür gezielt ins Land geholt. Polen spricht von einem »hybriden Krieg«, den Lukaschenko führt, um die Sta- } \\
\text { bilität Polens, aber auch Litauens sowie der EU zu erschüttern. }\end{array}$ \\
\hline
\end{tabular}




\begin{tabular}{|c|c|}
\hline 15.09 .2021 & $\begin{array}{l}\text { Die Pressesprecherin von Recht und Gerechtigkeit (Prawo i Sprawiedliwość - PiS), Anita Czerwińska, gibt } \\
\text { bekannt, dass der Politische Ausschuss der PiS einen Beschluss verabschiedet hat, in dem Polens Mitgliedschaft } \\
\text { in der Europäischen Union bekräftigt sowie seine staatliche Souveränität unterstrichen wird. Die PiS widerspre- } \\
\text { che damit den von der Opposition in Umlauf gebrachten Gerüchten, dass die PiS einen »Polexit« anstrebe. In } \\
\text { dem Beschluss wird außerdem die Notwendigkeit der Reformierung der EU festgestellt. Zu diesem Ziel hat die } \\
\text { PiS bereits Kontakt zu anderen Mitte-Rechts-Parteien in verschiedenen EU-Mitgliedsländern aufgenommen. }\end{array}$ \\
\hline 15.09 .2021 & $\begin{array}{l}\text { Bundesaußenminister Heiko Maas stellt in Berlin das Konzept für den »Ort des Erinnerns und der Begegnung } \\
\text { mit Polen« vor, wo an das von den Deutschen verursachte Leid der Polen während des Zweiten Weltkrieges } \\
\text { erinnert werden soll. Das gemeinsame Erinnern und die Vermittlung von historischem Wissen sowie Begeg- } \\
\text { nungen von Deutschen, Polen und den europäischen Nachbarn sollen im Mittelpunkt stehen. Der stellvertre- } \\
\text { tende Außenminister Szymon Szynkowski vel Sęk sagt auf einer Pressekonferenz, dass polnische Experten und } \\
\text { Betroffene bei den vorbereitenden Arbeiten bisher nicht ausreichend beteiligt worden seien. Sie sollten bei den } \\
\text { kommenden Schritten berücksichtigt werden. }\end{array}$ \\
\hline 17.09 .2021 & $\begin{array}{l}\text { Am zweiten Tag seines offiziellen Besuches wird Senatsmarschall Tomasz Grodzki in Berlin von Bundesrats- } \\
\text { präsident Reiner Haseloff empfangen. Zusammen mit einer Delegation nimmt Grodzki an einer Plenarsit- } \\
\text { zung teil. Grodzki sagt vor dem Bundesrat, der Senat der Republik Polen, in dem die Opposition die Mehrheit } \\
\text { hat, setze sich intensiv dafür ein, dass Polen im Kreise der westlichen Zivilisation bleibe, was vor dem Hinter- } \\
\text { grund der einstigen Zugehörigkeit zum sowjetischen Block von besonderer Bedeutung sei. Er informiert über } \\
\text { die Gründung einer deutsch-polnischen Gruppe im Senat. }\end{array}$ \\
\hline 18.09 .2021 & $\begin{array}{l}\text { In seiner Rede auf dem Parteitag der Bürgerplattform (Platforma Obywatelska-PO) in Płońsk fordert Partei- } \\
\text { chef Donald Tusk eine Verfassungsänderung. In Anlehnung an die bestehende Regelung, dass das Parlament } \\
\text { mit Zweidrittelmehrheit Kompetenzen der Regierung einer internationalen Organisation oder Organ über- } \\
\text { tragen kann, spricht sich Tusk dafür aus, in die Verfassung aufzunehmen, dass auch für die Auflösung eines } \\
\text { solchen Vertrags eine Zweidrittelmehrheit notwendig ist. So könne verhindert werden, dass das Regierungs- } \\
\text { lager von Recht und Gerechtigkeit (Prawo i Sprawiedliwość - PiS) den Austritt Polens aus der Europäischen } \\
\text { Union beschließt. }\end{array}$ \\
\hline 19.09 .2021 & $\begin{array}{l}\text { Der Grenzschutz teilt mit, dass auf polnischem Gebiet an der Grenze zu Belarus drei Leichen gefunden wur- } \\
\text { den. Es handele sich mutmaßlich um illegale Migranten, so Anna Michalska, Unterleutnant des Grenzschut- } \\
\text { zes. Die Staatsanwaltschaft habe die Ermittlungen aufgenommen. An der Grenze zwischen Belarus und Polen } \\
\text { kampieren seit Wochen Flüchtlinge u. a. aus dem Nahen Osten und Afghanistan, die der belarussische Prä- } \\
\text { sident Alexander Lukaschenko gezielt ins Land geholt und an die Westgrenze gebracht haben soll. Polen und } \\
\text { Litauen verweigern den Einlass, Belarus will sie nicht zurücknehmen. In Polen gilt seit Anfang September der } \\
\text { Ausnahmezustand im Grenzstreifen. }\end{array}$ \\
\hline 20.09 .2021 & $\begin{array}{l}\text { Auf einer Pressekonferenz gibt Ministerpräsident Mateusz Morawiecki bekannt, dass die drei am Vortag im pol- } \\
\text { nischen Grenzgebiet zu Belarus tot aufgefundenen Personen mutmaßlich an Unterkühlung und Erschöpfung } \\
\text { gestorben sind. Weitere geschwächte Personen seien in Krankenhäuser in der Nähe gebracht worden. Zurzeit } \\
\text { kampieren Flüchtlinge u. a. aus dem Nahen Osten und Afghanistan auf belarussischer Seite. Polen, Litauen } \\
\text { und die Europäische Union gehen davon aus, dass der belarussische Staatspräsident Alexander Lukaschenko } \\
\text { sie gezielt ins Land geholt hat, damit sie die Grenze zur EU übertreten können. Die EU-Staaten verweigern die } \\
\text { illegale Einreise und Belarus nimmt sie nicht zurück. Morawiecki sagt, seit August habe es an der polnisch- } \\
\text { belarussischen Grenze mehr als } 7.000 \text { Versuche illegaler Grenzübertritte nach Polen gegeben. }\end{array}$ \\
\hline 20.09 .2021 & $\begin{array}{l}\text { Der Europäische Gerichtshof (EuGH) verpflichtet Polen zu Strafzahlungen in Höhe von } 500.000 \text { Euro pro } \\
\text { Tag an die Europäische Kommission, da Polen den Braunkohleabbau im Tagebauwerk Turów trotz einer von } \\
\text { Tschechien im Februar eingereichten Klage sowie Antrag auf einstweilige Verfügung und einer entsprechen- } \\
\text { den Anordnung des EuGH am 21. Mai nicht eingestellt hat. Tschechien sieht den Zugang zum Grundwasser } \\
\text { im Raum Liberec durch den Tagebau gefährdet. Turów liegt in der Woiwodschaft Niederschlesien (wojewódz- } \\
\text { two dolnośląskie) im Dreiländereck und gehört zum Energiekonzern PGE, dessen Anteile mehrheitlich vom } \\
\text { polnischen Staat gehalten werden. }\end{array}$ \\
\hline
\end{tabular}

Sie können die gesamte Chronik seit 2007 auch auf http://www.laender-analysen.de/polen/ unter dem Link "Chronik« lesen. 
Die Polen-Analysen erscheinen zweimal monatlich als E-Mail-Dienst. Sie werden gemeinsam vom Deutschen PolenInstitut Darmstadt, der Forschungsstelle Osteuropa an der Universität Bremen und weiteren Partnern eines Konsortiums (siehe Titelseite) herausgegeben.

Ein Archiv der Polen-Analysen finden Sie im Internet unter www.laender-analysen.de/polen Kostenloses Abonnement unter http://www.deutsches-polen-institut.de/Newsletter/subscribe.php

Diese Analysen finden Sie online als Lizenzausgabe auf bpb.de

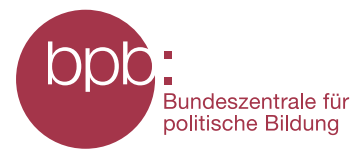

Deutsches Polen-Institut Darmstadt (www.deutsches-polen-institut.de)

Das seit 1980 tätige Deutsche Polen-Institut Darmstadt (DPI) ist ein Forschungs-, Informations- und Veranstaltungszentrum für polnische Kultur, Geschichte, Politik, Gesellschaft und die deutsch-polnischen Beziehungen, die sich im Kontext der europäischen Integration entwickeln. Institutionelle Träger des DPI sind das Land Hessen, die Kultusminister der Länder, das Auswärtige Amt und die Wissenschaftsstadt Darmstadt. Einen wesentlichen Beitrag zur Verwirklichung der Institutsziele leisten private Stiftungen. Ziel der Vermittlertätigkeit des DPI ist es, "die zu interessieren, auf die es politisch, wirtschaftlich, gesellschaftlich und kulturell im deutsch-polnischen Verhältnis ankommt (Leitlinien 1997). Es geht um die Entscheider und Multiplikatoren in Politik, Kultur, Bildung, Verwaltung, Medien und Wirtschaft. Das DPI versteht sich in Kooperation mit den Orten wissenschaftlicher Polen-Kompetenz an deutschen Hochschulen und Forschungsinstituten als verbindendes und vernetzendes Zentrum. Mit der 70.000 Bände zählenden multidisziplinären Fachbibliothek für Polen, die eine einzigartige Sammlung polnischer Belletristik in der Originalsprache und in deutscher Übersetzung umfasst, ist das DPI ein geschätzter Ort der Recherche und des wissenschaftlichen Arbeitens.

Forschungsstelle Osteuropa an der Universität Bremen (www.forschungsstelle.uni-bremen.de)

1982 gegründet, widmet sich die Forschungsstelle Osteuropa an der Universität Bremen der interdisziplinären Analyse der Länder Ost- und Ostmitteleuropas in Zeitgeschichte und Gegenwart. Der Forschungsschwerpunkt liegt dabei auf der Rolle von "Dissens und Konsens«, von Opposition und Zivilgesellschaft in ihrem historischen, politischen, gesellschaftlichen und kulturellen Kontext. Die Forschungsstelle besitzt in ihrem Archiv eine einzigartige Sammlung alternativer Kulturgüter und unabhängiger Texte aus den ehemaligen sozialistischen Ländern. Darunter befindet sich auch eine umfangreiche Sammlung des "Zweiten Umlaufs«, die das Schrifttum und Dokumente unabhängiger Initiativen und gesellschaftlicher Gruppen in Polen aus der Zeit von 1976 bis zum Umbruch umfasst. Hinzu kommt eine umfangreiche Bibliothek mit wissenschaftlicher Literatur. Mit Archiv, Bibliothek und zwei wissenschaftlichen Abteilungen ist die Forschungsstelle auch eine Anlaufstelle sowohl für Gastwissenschaftler als auch für die interessierte Öffentlichkeit.

Eine der Hauptaufgaben der Forschungsstelle ist die Information der interessierten Öffentlichkeit. Dazu gehören unter anderem regelmäßige E-Mail-Informationsdienste für Politik, Wirtschaft, Zivilgesellschaft und Medien.

Herausgeber:

Deutsches Polen-Institut, Deutsche Gesellschaft für Osteuropakunde e.V., Forschungsstelle Osteuropa an der Universität Bremen, Leibniz-Institut für Agrarentwicklung in Transformationsökonomien, Leibniz-Institut für Ost- und Südosteuropaforschung, Zentrum für Osteuropa- und internationale Studien (Z0iS) gGmbH

Redaktion:

Dr. Andrzej Kaluza (verantwortlich) (Darmstadt) und Dr. Silke Plate (Bremen)

Satz: Matthias Neumann

Wissenschaftlicher Beirat:

Prof. Dr. Stefan Garsztecki, Technische Universität Chemnitz

Prof. Dr. Klaus Ziemer, Kardinal-Stefan-Wyszyński-Universität Warschau

Die Meinungen, die in den Polen-Analysen geäußert werden, geben ausschließlich die Auffassung der Autoren wieder Abdruck und sonstige publizistische Nutzung sind nach Rücksprache mit der Redaktion gestattet. Polen-Analysen-Layout: Cengiz Kibaroglu, Matthias Neumann

Alle Ausgaben der Polen-Analysen sind mit Themen- und Autorenindex archiviert unter www.laender-analysen.de

ISSN 1863-9712 @ 2021 by Deutsches Polen-Institut, Deutsche Gesellschaft für Osteuropakunde e.V., Forschungsstelle Osteuropa an der Universität Bremen, Leibniz-Institut für Agrarentwicklung in Transformationsökonomien, Leibniz-Institut für Ost- und Südosteuropaforschung, Zentrum für Osteuropa- und internationale Studien (ZOiS) gGmbH Kontakt: Dr. Andrzej Kaluza, Deutsches Polen-Institut, Residenzschloss, Marktplatz 15, 64283 Darmstadt,

Tel.: +49/6151/4202-20, Fax: +49/6151/4202-10, E-Mail: kaluza@dpi-da.de, Internet: www.laender-analysen.de/polen 


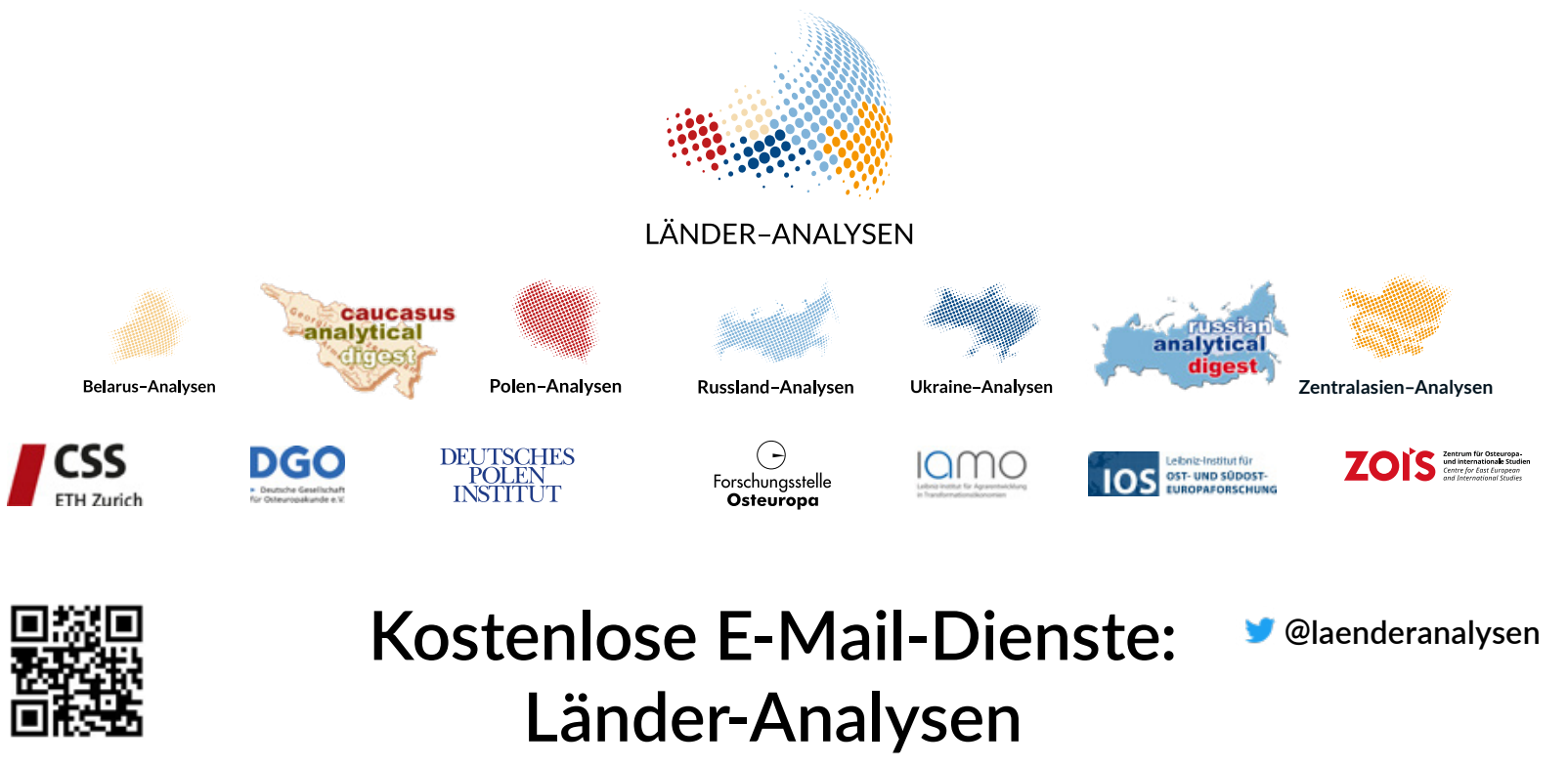

Die Länder-Analysen bieten regelmäßig im kostenlosen Abonnement kompetente Einschätzungen aktueller politischer, wirtschaftlicher, sozialer und kultureller Entwicklungen in Ostmitteleuropa und der GUS. Alle Länder-Analysen verstehen sich als Teil eines gemeinsamen Projektes, das der wissenschaftlich fundierten, allgemeinverständlich formulierten Analyse der Entwicklungen im östlichen Europa, der Offenheit für verschiedene inhaltliche Positionen und der kostenlosen und nicht-kommerziellen Information einer breit verstandenen interessierten Öffentlichkeit verpflichtet ist. Autor/innen sind internationale Fachwissenschaftler/innen und Expert/innen. Die Redaktionen der Länder-Analysen bestehen aus Wissenschaftler/innen mit langjähriger Forschungserfahrung.

Die deutschsprachigen Länder-Analysen werden gemeinsam von der Forschungsstelle Osteuropa an der Universität Bremen, dem Zentrum für Osteuropa- und internationale Studien, der Deutschen Gesellschaft für Osteuropakunde, dem Deutschen Polen-Institut, dem Leibniz-Institut für Agrarentwicklung in Transformationsökonomien und dem Leibniz-Institut für Ost- und Südosteuropaforschung herausgegeben. Die englischsprachigen Länder-Analysen erscheinen in Kooperation der Forschungsstelle Osteuropa mit dem Center for Security Studies (CSS) der ETH Zürich.

Die Länder-Analysen bieten regelmäßig Kurzanalysen zu aktuellen Themen, ergänzt um Grafiken und Tabellen sowie Dokumentationen. Zusätzlich gibt es eine Chronik aktueller Ereignisse.

\section{Belarus-Analysen}

Erscheinungsweise: zweimonatlich

Abonnement unter: http://www.laender-analysen.de/belarus/

\section{Caucasus Analytical Digest}

In englischer Sprache. Erscheinungsweise: zweimonatlich

Abonnement unter: http://www.css.ethz.ch/en/publications/cad.html

\section{Polen-Analysen}

Erscheinungsweise: zweimal monatlich

Abonnement unter: http://www.deutsches-polen-institut.de/newsletter/polen-analysen/

\section{Russland-Analysen}

Erscheinungsweise: zweimal monatlich

Abonnement unter: http://www.laender-analysen.de/russland/

\section{Russian Analytical Digest}

In englischer Sprache. Erscheinungsweise: zweimal monatlich Abonnement unter: http://www.css.ethz.ch/en/publications/rad.html

\section{Ukraine-Analysen}

Erscheinungsweise: zweimal monatlich

Abonnement unter: http://www.laender-analysen.de/ukraine/

\section{Zentralasien-Analysen}

Erscheinungsweise: zweimonatlich

Abonnement unter: http://www.laender-analysen.de/zentralasien/ 